\title{
Effect of Surface Mechanical Attrition Treatment on Micro-mechanical Properties of ZrCuAINi Bulk Metallic Glass
}

\author{
Yerzhan Shayakhmetov ${ }^{a}$, Alla Vorobeva ${ }^{b}$, Stepan Burlankov', Konstantin Bogonosov ${ }^{b}$, Andrei Fomin ${ }^{\text {, }}$ \\ Andrey Goncharov ${ }^{b}$, Stepan Krasnikov ${ }^{b}$, Svetlana Nikolaeva ${ }^{e}$, Anna Ovsyannikova ${ }^{b}$, \\ Angelina Olegovna Zekiyf, Mahander Pandey ${ }^{{ }^{*}}$ (D) \\ a Shakarim University of Semey, Semey, Kazakhstan. \\ ${ }^{b}$ K. G. Razumovsky Moscow State University of Technologies and Management (The First Cossack \\ University), Moscow, 109004, Russian Federation. \\ cPlekhanov Russian University of Economics, Moscow, 117997, Russian Federation. \\ ${ }^{d}$ National Research Mordovia State University, Mordovia, 430005, Russian Federation. \\ ${ }^{e}$ MIREA - Russian Technological University, Moscow, 119454, Russian Federation. \\ ${ }^{f}$ Sechenov First Moscow State Medical University, Moscow, 119991, Russia Federation. \\ ${ }^{g}$ Indian Institute of Technology Heydarabad, Department of Materials Science and Metallurgical \\ Engineering, Heydarabad, 502258, India.
}

Received: March 01, 2021; Revised: June 28, 2021; Accepted: June 30, 2021

In this work, surface mechanical attrition treatment (SMAT) was employed to rejuvenate $\mathrm{ZrCuAlNi}$ bulk metallic glass (BMG) plate. Differential scanning calorimetry (DSC), atomic force microscopy (AFM) and nanoindentation analyses were carried out to evaluate stored energy and micro-mechanical properties of treated BMGs. According to DSC results, 10 min SMAT process increased the stored energy of BMG plates up to 50\%. AFM analysis showed that the structural rejuvenation occurred in the bulk of samples and just a slight rejuvenation gradient was detected from the front to the back side of BMG plates. Nanoindentation analysis indicated that the structural rejuvenation is consistent with anelastic strain induced under the SMAT process. It was also found that an optimum treatment time is needed for maximum rejuvenation in the BMGs. This event is due to the fact that the glassy structure is able to store a critical anelastic strain, which leads to a saturated condition in rejuvenation.

Keywords: Bulk metallic glass, rejuvenation, relaxation, nanoindentation, heterogeneity.

\section{Introduction}

Due to their outstanding mechanical and physical properties, bulk metallic glasses (BMGs) have received tremendous research interest for developing novel engineering structures $^{1-3}$. However, practical application of BMGs have been hindered in many cases, owing to their inhomogeneous plastic deformation ${ }^{4,5}$. To tackle this challenge, several methods such as chemical composition tuning, composite making or rejuvenating processes have been proposed ${ }^{6-10}$. Among them, rejuvenation process is an applicative technique, in which structural atomic excitation occurs and glassy material moves to higher energy state ${ }^{11-16}$. Thanks to many rejuvenation processes proposed by researchers, mechanical treatments in the range of elastic and anelastic deformation are identified effective methods, in which no shape changes occur and there is no limit in dimensions of samples. For example, Zhang et al. ${ }^{17}$ found that $\mathrm{ZrTiBeCu}$ BMG shows a relaxation-to-rejuvenation under compressive elastostatic loading. It was also reported that the compressive loading is effective near the range of yield strength. In the other works, Samavatian et al. ${ }^{18-20}$ reported that the tensile

*e-mail: Pandey.mahander1@gmail.com elastostatic loading at low stress levels can rejuvenate the atomic structure. Using pair distribution function analysis, they revealed that the elastostatic loading markedly decreases short range order (SRO) and medium range order (MRO) in the atomic configuration. Tong et al. ${ }^{21}$ determined that thermo-mechanical creep processes under an anelastic deformation range enhances the disordering event in BMGs. Using molecular dynamic (MD) simulation of cyclic loading, it was found that rejuvenation is correlated with the increase in nanoscale heterogeneity and, nucleation and distribution of shear transformation zones ${ }^{22,23}$. Using shock stress amplitudes, Ding et al. ${ }^{24}$ successfully rejuvenated $\mathrm{Zr}_{55} \mathrm{Cu}_{30} \mathrm{Ni}_{5} \mathrm{Al}_{10}$ on very short time scales. In another study, Sohrabi et al. ${ }^{25}$ indicated that an intense ultrasonic elastic processing may be lead to the increased atomic mobility and oscillatory energy storage.

Considering to the literature, development of new mechanical methods is very crucial to enhance structural rejuvenation and plasticity of BMGs. For this purpose, in this work we used a mechanical processing, namely surface mechanical attrition treatment (SMAT), to make more capabilities for characteristic improvement of a Zr-based BMG plate under a structural rejuvenation evolution. In SMAT process, a 
severe plastic deformation occurs at high energy, inducing nano-crystalline phases at surface layer of a crystalline materials ${ }^{26}$. Applying SMAT for BMGs, it was found that gradient nano-crystalline structure may be formed if the impact energy becomes much enough ${ }^{27}$. Hence, in this work we tune the impact energy somehow to rejuvenate our BMG without formation of any crystalline phase in the structure.

\section{Materials and Methods}

$\mathrm{Zr}_{55} \mathrm{Cu}_{30} \mathrm{Al}_{10} \mathrm{Ni}_{5}$ (at. \%) ingots were prepared using high purity elements (>99.98\%) under a Ti-gettered Ar atmosphere arc-melting technique. The real chemical composition after arc-melting process was $\mathrm{Zr}_{55.14} \mathrm{Cu}_{29.95} \mathrm{Al}_{9.74} \mathrm{Ni}_{5.17}$ (at. \%). The prepared ingots were then cast into plates with dimensions of $2 \times 20 \times 20 \mathrm{~mm}^{3}$ by a water-cooled copper mold casting process. After fabrication of plates, the X-ray diffraction (XRD) analysis with $\mathrm{CuK} \alpha$ radiation $(1.5418 \AA)$ was done to confirm the amorphous nature of BMG. The BMG samples were then exposed to surface mechanical attrition treatment (SMAT). For this process, a $15 \mathrm{KHz}$ ultrasonic transducer was applied at room temperature to excite hard spherical shots with $1 \mathrm{~mm}$ diameter. The spherical shots impacted on the surface of samples for 5, 10 and $15 \mathrm{~min}$. To determine the conditions of samples in our study, the as-cast and treated samples were coded as S0, S5, S10 and $\mathrm{S} 15$, respectively. As given in Figure 1a, it is found that the BMGs remained amorphous after SMAT process. Moreover, the structural nature of samples S0 and S15 was evaluated by transmission electron microscopy (TEM- FEI Titan G2). For this purpose, the samples were prepared in the form of thin foils using ion milling technique under a liquid-nitrogen cooling procedure. As illustrated in Figure 1b, the TEM images appear in a salt-and-pepper contrast for both of the samples. The diffraction patterns also show diffuse halo rings, implying on the absence of any crystalline phases in the as-cast and the treated states.

For measuring enthalpy of structural relaxation $\left(\Delta \mathrm{H}_{\text {rel }}\right)$, differential scanning calorimetry (DSC) was performed under argon environment and heating/cooling rate of $20 \mathrm{~K} / \mathrm{min}$. In order to calculate $\Delta \mathrm{H}_{\text {rel }}$, the samples were slightly heated beyond the glass transition temperature ${ }^{28}$. The heated samples were then cooled to the room temperature and again heated with the same heating rate $(20 \mathrm{~K} / \mathrm{min})$ to the same temperature. Subtracting the second thermograph from the first one, it is possible to obtain the $\Delta \mathrm{H}_{\text {rel }}$, which is related to the area of relaxation under the glass transition temperature. Three samples were analyzed in the DSC experiment for each state. Using Bruker atomic force microscope (AFM), the local dynamic modulus was measured for each sample. Before performing the experiment, the samples were polished carefully to obtain a smooth surface with roughness of 2-3 nm. Tip radius was 5-7 $\mathrm{nm}$ and subsequently the maximum applied force was $250 \mathrm{nN}$, which is in the range of BMG's elastic limit. A surface area of $800 \times 800 \mathrm{~nm}$ was evaluated for each sample. Dynamic modulus was calculated according to the Sneddon cone-on-flat model ${ }^{29}$. For nanoindentation evaluation, the experiment was conducted by Anton Paar NHT instrument under an applied load of $30 \mathrm{mN}$, loading rate of $5 \mathrm{mN} / \mathrm{mm}$ and holding time of $3 \mathrm{~S}$ at the maximum load. To have statistical evaluation, data of 60 indenting points were collected for each specimen. The magnitude of derivative $\left(d h / d p^{0.5}\right)$, showing the variations of pop-in events with respect to applied load (p), was also calculated. Moreover, scanning electron microscopy (SEM- TESCAN vega2) was used to detect the propagation of shear bands in the nanoindentation zone.

\section{Results}

It is firstly necessary to show that how much SMAT process affects the enthalpy of structural relaxation in the Zr-based BMG. Figure 2 illustrates magnified DSC curves of as-cast and treated samples at the relaxation region below glass transition temperature $\left(\mathrm{T}_{\mathrm{g}}\right)$. Moreover, Table 1 presents the average values of thermal features for each state. As observed in Figure 2, as-cast sample (S0) has $4.95 \mathrm{~J} / \mathrm{g}$ enthalpy of relaxation, while it was measured about 6.4, 7.3 and $5.3 \mathrm{~J} / \mathrm{g}$ for samples S5, S10 and S15, respectively. This result clearly indicates that the SMAT process leads to the increased stored energy, especially at the treatment time of 5 and $10 \mathrm{~min}$; however, at the higher
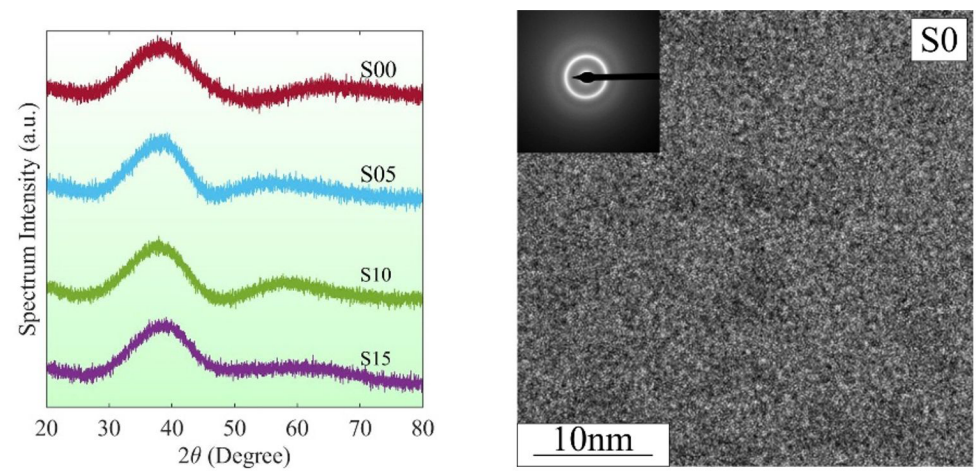

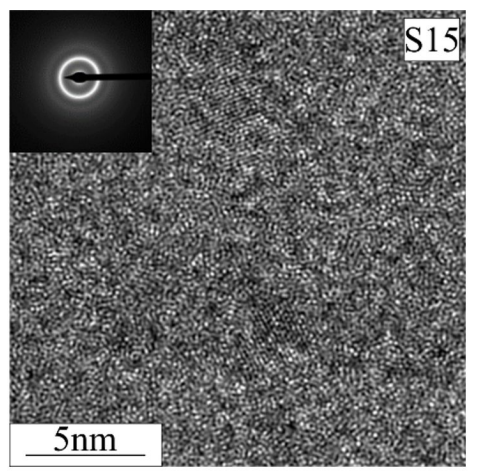


treatment times (15 $\mathrm{min})$, rejuvenation degree decreases, which means that an optimum treatment time is needed for maximum structural rejuvenation in the BMG. Figure 3 shows the statistical variations of dynamic modulus in the treated samples at the front side (impacted regions) and the back side. As given in Figure 3a, the SMAT process sharply decreases the intensification of dynamic modulus and extends the statistical distribution, which implies that the structural heterogeneity increases after the treatment. In other words, when the heterogeneity increases in the material, nanoscale regions with a wide range of elastic properties appear in the structure $^{18}$. Hence, one can see that statistical distribution broadens and also a decrease in sharpness of spectrum was observed, which confirms that the defects, i.e. disordered

Table 1. Average values of thermal features in each state.

\begin{tabular}{ccccc}
\hline & $\mathrm{T}_{\mathrm{g}}(\mathrm{K})$ & $\mathrm{T}_{\mathrm{x}}(\mathrm{K})$ & $\Delta \mathrm{H}_{\text {rel }}(\mathrm{J} / \mathrm{g})$ & $\Delta \mathrm{Q}(\mathrm{J} / \mathrm{g})$ \\
\hline $\mathrm{S} 00$ & $694 \pm 2.5$ & $746.4 \pm 1$ & $4.94 \pm 0.35$ & $104 \pm 3$ \\
\hline $\mathrm{S} 05$ & $691 \pm 2$ & $745.3 \pm 1.5$ & $6.36 \pm 0.27$ & $105.5 \pm 2.5$ \\
\hline $\mathrm{S} 10$ & $691.3 \pm 3$ & $742 \pm 1.8$ & $7.34 \pm 0.31$ & $105.7 \pm 3.1$ \\
\hline $\mathrm{S} 15$ & $692 \pm 1.5$ & $748.2 \pm 2.2$ & $5.32 \pm 0.41$ & $108 \pm 3.3$ \\
\hline
\end{tabular}

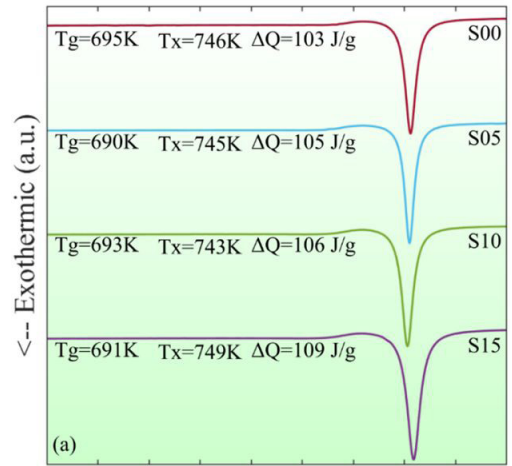

$400 \quad 450500550600 \quad 650 \quad 700 \quad 750 \quad 800 \quad 850$ Temperarure $(\mathrm{K})$

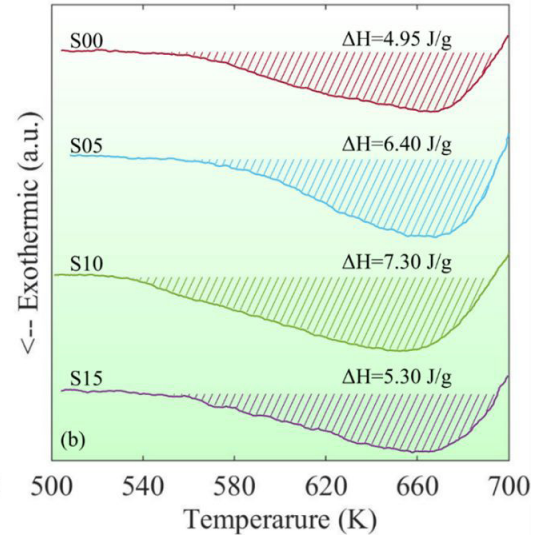

Figure 2. a) DSC curves of samples and b) magnified relaxation region in DSC curves
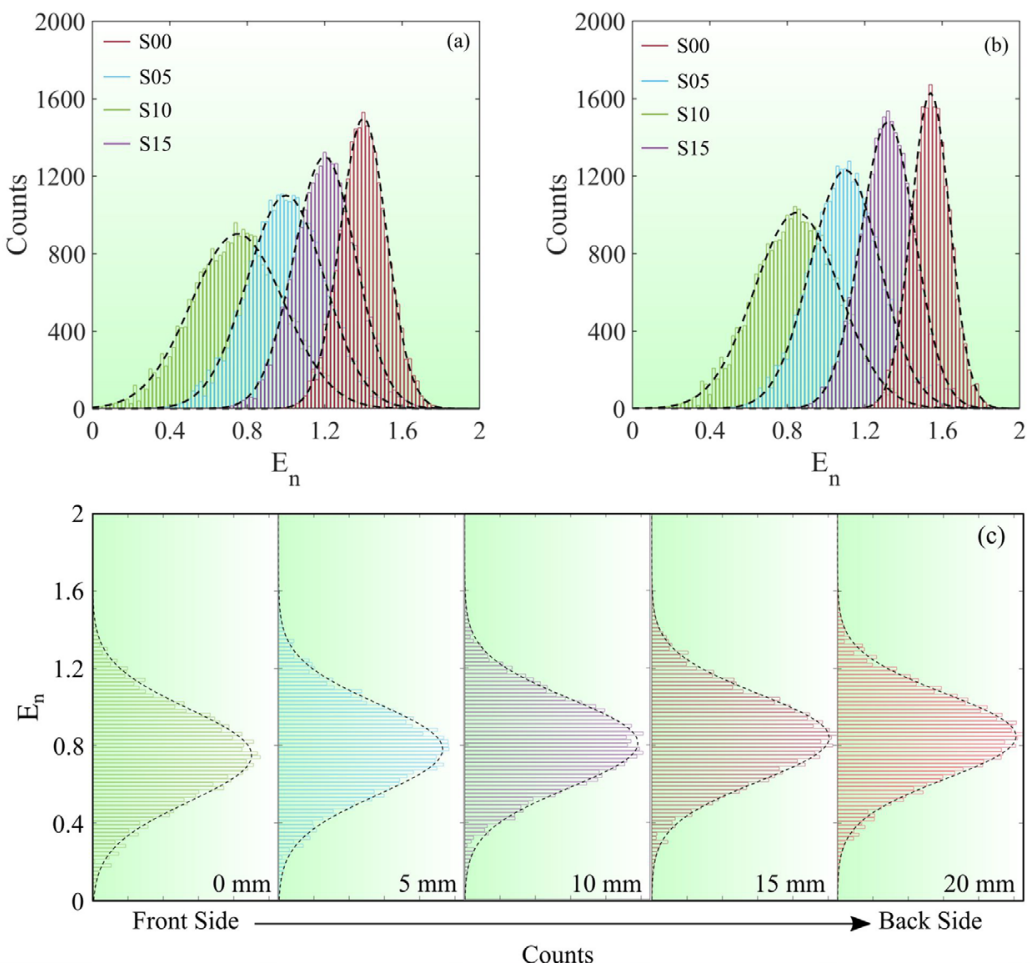

Figure 3. normalized dynamic modulus for BMG samples at a) front side and b) back side. c) Violin plot of the normalized dynamic modulus in the depth of sample S10 from front side into the back side. Dynamic modulus was normalized by the lowest measured value in the test. 
structures such as free volumes, are increased after the SMAT treatment. Zhu et al. ${ }^{30}$ indicated that the relation between amorphous structure and its dynamic behavior reveals the mechanism of $\beta$ relaxation in the MGs. under the AFM experiment, no macroscopic deformation happens in the MGs and the atomic rearrangements restricted to the nanoscale cooperative motions and short-range diffusion phenomenon, which is sign of structural relaxation and the change of relaxation enthalpy in the amorphous alloys. This event is observed in the form of local frustration-limited domains or the spatial heterogeneity in the map of energy dissipation ${ }^{18,30}$ or change in the distribution of elastic response of glassy structure, which is also seen in our work.

Figure $3 \mathrm{~b}$ indicates dynamic modulus distribution measured at the back side of BMG plates. As observed, the trend of results is similar to what measured at the front side of BMGs. However, a slight discrepancy is seen between dynamic modulus distribution at the front and back sides. Totally it is concluded that the SMAT process is not restricted at the surface of BMGs and the whole body of BMG plate is affected from the treatment, so that the structural rejuvenation occurs in the bulk of material. With all these descriptions, the results suggest that a slight rejuvenation gradient may be seen in the thickness of BMGs. To have a precise analysis of rejuvenation gradient in the treated alloy, we performed the AFM test in the depth of sample S10. For this purpose, the AFM was carried out at the front side, i.e. $0 \mathrm{~mm}, 0.5 \mathrm{~mm}$, $1 \mathrm{~mm}, 1.5 \mathrm{~mm}$ and the back side, i.e. $2 \mathrm{~mm}$. To reach the depth of sample for the AFM analysis, a polishing process was done to decrease the thickness to the certain values. As given in Figure 3c, the move into the depth of sample S10 from the front side to the back side is accompanied with the contraction and intensification of $\mathrm{E}_{\mathrm{n}}$ distribution, indicating the slight rejuvenation gradient and the change in the rate of heterogeneity in the glassy structure.

Figure $4 \mathrm{a}$ gives the load-displacement curves of BMG samples. It is detected that the SMAT process leads to the considerable enhancement of displacement, compared to the as-cast situation. There are also critical parameters describing the deformation behavior under the nanoindentation test. For example, final indentation depth $\left(\mathrm{d}_{\mathrm{f}}\right)$ and maximum indentation depth $\left(\mathrm{d}_{\max }\right)$ are denoted in Figure $4 \mathrm{a}$. The total creep displacement at $d_{\text {max }}$ of curves shows an increasing trend under the SMAT process; however, again an optimum value is measured for the creep displacement at $10 \mathrm{~min}$ SMAT process (inset Figure 4a). In general, the increased creep is correlated to the anelastic and viscoplastic strains induced under the indentation. Considering Figure $4 b$, it is found that the $d_{f} / d_{\max }$ rises with the evolution of structural rejuvenation in our BMG alloy. This means that the rate of $d_{f}$ enhancement is significantly higher than $d_{\max }$ for the rejuvenated samples. The bigger $\mathrm{d}_{\mathrm{f}}$ is closely related to this fact that more anelastic energy is stored in the BMG structure. Hence, one can concluded that the structural rejuvenation under SMAT process strongly depends on anelastic strains, which is also consistent with that was reported in other works $^{21,31}$. As mentioned, an optimum structural rejuvenation occurs under the SMAT process (S10). So it is derived that the potential of BMG structure for storing anelastic strain energy is the main factor defining the rejuvenation level. In order to understand the total creep variations under the nanoindentation test, it is required to statistically evaluate Young's modulus and hardness of BMGs. Figure 5 shows the relation between total creep, hardness and Young's modulus for the as-cast and treated samples. The results accurately unveiled that the hardness and Young's modulus dramatically decrease after the SMAT process so that the average hardness and Young's modulus for sample S10 is 16\% and 23\% lower than the as-cast sample (S0), respectively. Moreover, trends of hardness and Young's modulus distribution show linear shapes with negative slopes of -0.15 and $-2.81 \mathrm{~nm} . \mathrm{GPa}^{-1}$ and statistical Pearson correlation coefficient of -0.817 and -0.864 respectively. The similarities in trend of hardness and Young's modulus distribution is due to the fact that the hardness and elastic properties show a linear correlation in the rejuvenated $\mathrm{BMGs}^{32}$. Compared to sample $\mathrm{S} 0$, it is also manifested that the rejuvenated samples have more extended statistical distribution, which is due to the induced structural heterogeneity after the treatment.

Figure 6 illustrates the measured pop-in displacements $(\Delta \mathrm{h})$ and the magnitude of derivative $\left(d h / d p^{0.5}\right)$, which are obtained from the load-displacement curves. Considering Figure 6, it is evolved that the small pop-in events generally tend to show low derivatives, while bigger pop-ins cause larger derivatives. Furthermore, one can find that rejuvenation leads to the contraction of data in both plots, which indicates that the rejuvenated $\mathrm{BMG}$ tends to the formation of multiple
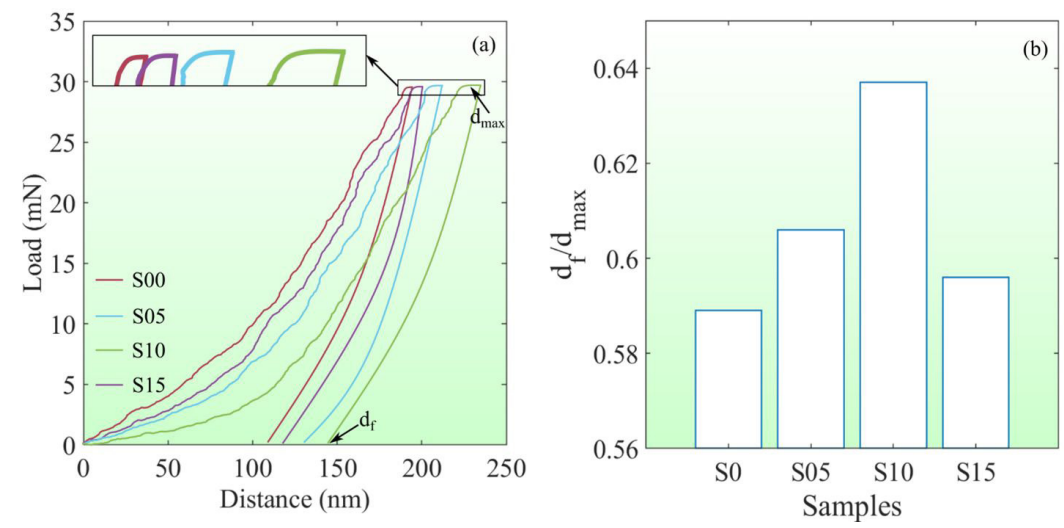

Figure 4. a) load-displacement curves for BMG samples, b) $d_{\mathrm{f}} / d_{\max }$ ratio extracted from load-displacement curves. 
small shear bands under the nanoindentation test ${ }^{33}$. In general, the shear bands are resulted from the activation and percolation of shear transformation zones (STZs) in a certain orientation $^{34,35}$, which is related to the applied stress fields in the system. In a rejuvenated structure, the enthalpy of relaxation increases which means that the concentration of flow units enhances in the glassy structure. In this condition, the structural heterogeneity is intensified and leads to the rise in fluctuations of stress fields in atomic scales ${ }^{36}$. Therefore, under an external force such as nanoindentation test, numerous number of shear embryo are activated in the bulk of material of rejuvenated samples and consequently the shear banding event occurs in multiple mode. The SEM micrographs with high magnification from the nanoindentation zones of S0 and S10 are shown in Figure 7. The images confirmed that the SMAT process leads to the formation of shear bands with significantly higher population and smaller sizes, which is consistent with the nanoindentation results.

\section{Discussion}

As an energy-level point of view, structural rejuvenation brings the glassy alloy into higher energy states, where the defects such as free volumes are excessively induced in the system $^{23}$. Moreover, structural rejuvenation is accompanied with a significant decline in hardness and Young's modulus and
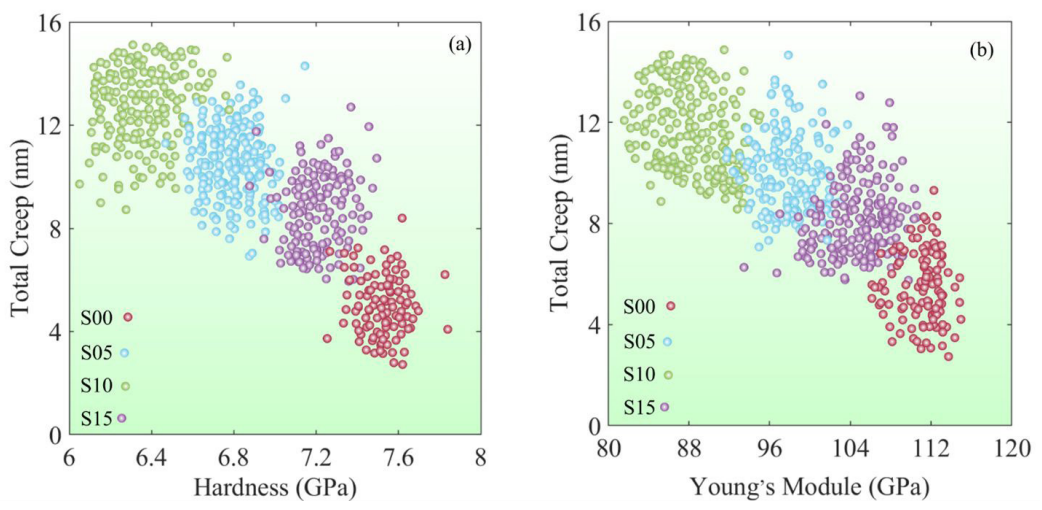

Figure 5. distribution of a) hardness and b) Young's modulus as a function of total creep in BMG samples.
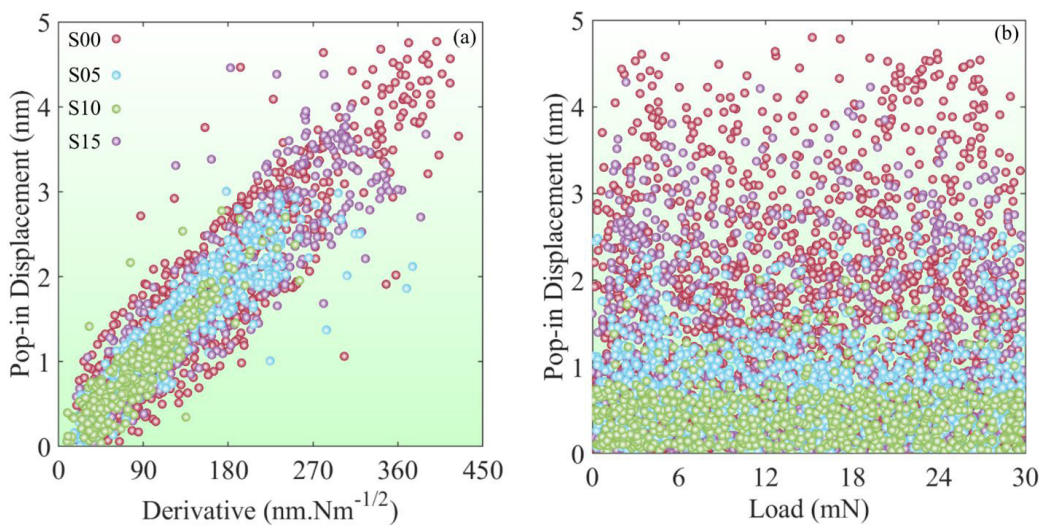

Figure 6. a) pop-in derivatives and b) load variations as function of pop-in displacements.
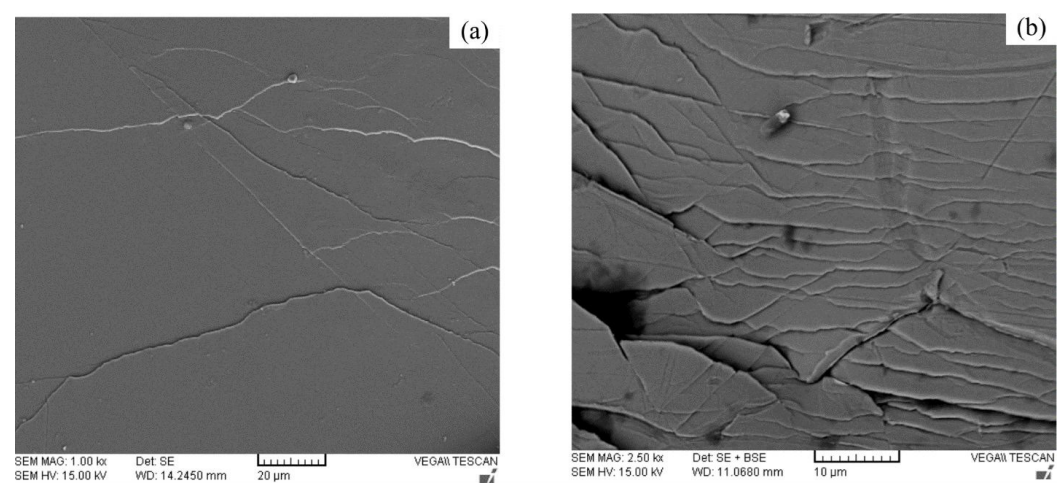

Figure 7. SEM micrographs of shear band propagation in a) S0 and b) S10 from the nanoindentation zones. 
an increase in relaxation enthalpy ${ }^{29}$. Here we should establish a meaningful correlation between our experimental results to show evolution of rejuvenation under the SMAT process.

In general, at low amplitude cycling or oscillatory excitations such as dynamic mechanical analysis (DMA), the induced strain is insignificant and consequently the glassy alloy shows a linear viscoelastic behavior. On the other side, the higher intensification of excitation, in which a considerable anelastic strain is induced into the system, leads to the nonlinear viscoelastic behavior of BMGs with a significant deviation from the linear elasticity ${ }^{32}$. In SMAT process, the stress level is high enough to induce a considerable nonlinear viscoelastic energy into the system. In many rejuvenation cases such as cryogenic cycling or high pressure torsion, it was found that the stored energy or strain softening under the rejuvenation treatment has a gradient behavior in the bulk of material ${ }^{29,32}$. This event may cause a sharp gradient of structural characteristics or mechanical properties over the bulk of BMG alloy. Under the SMAT process, it was found that an average slight gradient of $8 \%$ for dynamic modulus exists in the thickness of our BMG plate, which means that the SMAT process is able to induce structural rejuvenation bulk glassy alloys (see Figure 3 ).

Considering creep deformation under nanoindentation test (inset Figure 4), it is observed that the rejuvenated structure strongly affects the plasticity in the BMGs. Moreover, it was generally accepted that the plasticity of glassy alloys inherently relies on relaxation enthalpy ${ }^{31,32}$. Based on the dynamics of relaxation events, total enthalpy of relaxation is divided into primary $(\alpha)$ relaxation adjacent to $T_{g}$ and a secondary $(\beta)$ relaxation ${ }^{28}$. The activation energy of $\beta$ relaxation is correlated to the activation of STZs, leading to an anelastic strain with nanoscale local deformation in the glassy structure. On the other side, the energy of $\alpha$ relaxation is consistent with the STZ percolation and large scale irreversible atomic rearrangements ${ }^{32}$. Due to merged part of relaxation in DSC curves, separating $\alpha$ and $\beta$ relaxation processes are almost impossible in many $\mathrm{MG}$ compositions. However, from final indentation depth $\left(\mathrm{d}_{\mathrm{f}}\right)$ measurements in the load-displacement curves of samples (see Figure 4), one can conclude that the structural rejuvenation is accompanied with larger $d_{f}$, showing the higher stored anelastic strain energy. Hence, it is found that the structural rejuvenation under SMAT process is consistent with the increase in formation and distribution of STZs in the material. Considering AFM results, one finds that the distribution of elastic modulus in rejuvenated samples are much higher than the as-cast one. This result confirms that the structural heterogeneity under rejuvenation is intensified and nanoscale regions with different elastic properties are increased. This event, which is caused by formation and distribution of STZs, leads to the multiple small shear events and a homogenous deformation under the nanoindentation process. The last question should be answered is why the rejuvenation degree decreases after 10 min SMAT process? Similar to our study, there are also some works which reported optimum parameters for maximum rejuvenation degree in the $\mathrm{BMGs}^{17,20}$. To give some examples, the rejuvenation processes such as cryogenic cycling or elastostatic loadings are also associated to the saturated anelastic strain with optimized parameters ${ }^{17,21}$.
In this study, the maximum enthalpy of relaxation was attained in the sample treated for $10 \mathrm{~min}$, while a decrease in the enthalpy was seen in the 15-min treated one. This shows that an optimized concentration of defects, such as free volumes, are generated in the glassy structure and then it decreases when the SMAT time increases from a certain value. In fact, a treatment in a MG alloy includes both of structural relaxation and rejuvenation ${ }^{19}$. When the structural rejuvenation reaches its maximum rate, a saturation of defect generation occurs, which is detected in S10. However, with the increase in SMAT time to $15 \mathrm{~min}$, the rate of structural relaxation outweighs the rejuvenation, which leads to the decrease in the stored energy in this sample. Moreover, the decrease in stored energy of S15 is accompanied with the contraction of dynamic modulus distribution, compared with S10. It is also should be noted that the decrease in the generation of defects and stored energy in S15 leads to the enhancement of hardness and Young's modulus than that analyzed in S10. From these results, it is concluded that the increase in stored energy in the amorphous solids is directly related to the rise in structural defects, heterogeneity and distribution of dynamic modulus, while it has an inverse correlation with the hardness and Young's modulus, which is also reported in other works ${ }^{18,29,37-40}$. Finally, it should be noted that the SMAT time was in the range of 0 up to $15 \mathrm{~min}$ with 5-min intervals. Consequently, one can conclude that the shortening of intervals may lead to the slight change in the optimization time of SMAT process for reaching maximum structural rejuvenation in the BMG.

\section{Summary}

For rejuvenating $\mathrm{ZrCuAlNi}$ (BMG) plates, SMAT was applied under different treatment times. The results showed that the enthalpy of structural relaxation increased from $4.95 \mathrm{~J} / \mathrm{g}$ to $7.3 \mathrm{~J} / \mathrm{g}$ after $10 \mathrm{~min}$ SMAT process. The AFM and nanoindentation tests indicated that maximum structural rejuvenation occurs at an optimum treatment time (10 min) and with the increase in time, the stored energy in the glassy structure tends to decrease. It is suggested that the structural rejuvenation is strongly correlated to the induced anelastic strain and STZ formation under SMAT process. Therefore, it is concluded that attaining optimum structural rejuvenation is consistent with the saturation of anelastic strain in the BMG. Moreover, it is observed that SMAT process is not restricted to the surface of material and it is able to induce rejuvenation into the thickness of BMG.

\section{References}

1. Schroers J. Processing of bulk metallic glass. Adv Mater. 2010;22(14):1566-97. http://dx.doi.org/10.1002/adma.200902776.

2. Wang WH. Dynamic relaxations and relaxation-property relationships in metallic glasses. Prog Mater Sci. 2019;106:100561. https://doi.org/10.1016/j.pmatsci.2019.03.006.

3. Zhang L-C, Jia Z, Lyu F, Liang S-X, Lu J. A review of catalytic performance of metallic glasses in wastewater treatment: recent progress and prospects. Prog Mater Sci. 2019;105:100576. https://doi.org/10.1016/j.pmatsci.2019.100576.

4. Qu R, Tönnies D, Tian L, Liu Z, Zhang Z, Volkert CA. Sizedependent failure of the strongest bulk metallic glass. Acta Mater. 2019;178:249-62. https://doi.org/10.1016/j.actamat.2019.08.019. 
5. Shi Z, Li R, Li X, Wang C, Zhang T. Controllable brittleness in soft-magnetic Fe-P-C-B metallic glasses through composition design. Mater Sci Eng A. 2019;766:138385. https://doi. org/10.1016/j.msea.2019.138385.

6. Zhang L, Narayan RL, Fu HM, Ramamurty U, Li WR, Li $\mathrm{YD}$, et al. Tuning the microstructure and metastability of $\beta$-Ti for simultaneous enhancement of strength and ductility of Ti-based bulk metallic glass composites. Acta Mater. 2019;168:24-36. https://doi.org/10.1016/j.actamat.2019.02.002.

7. Chen S-Q, Hui K-Z, Dong L-Z, Li Z, Zhang Q, Gu L, et al. Excellent long-term reactivity of inhomogeneous nanoscale Fe-based metallic glass in wastewater purification. Sci. China Mater. 2020;63:453-66. http://dx.doi.org/10.1007/s40843-0191205-5.

8. Lin S, Zhu Z, Ge S, Zhang L, Liu D, Zhuang Y, et al. Designing new work-hardenable ductile Ti-based multilayered bulk metallic glass composites with ex-situ and in-situ hybrid strategy. J Mater Sci Technol. 2020;50:128-38. https://doi.org/10.1016/j. jmst.2019.12.037.

9. Anatolyevna KI, Yulia Vladimirovna F, Vladimirovna BO, Anatolyevna BO, Ivanovna GM, Alexandrovna KT. Impact of ultrasonic treatment on physicochemical properties of polyethylene compositions containing silver nanoparticles. Eurasian Chem. Commun. 2020;2:1126-36.

10. Louzguine-Luzgin DV, Jiang J, Bazlov AI, Zolotorevzky VS, Mao $\mathrm{H}$, Ivanov $\mathrm{YP}$, et al. Phase separation process preventing thermal embrittlement of a Zr-Cu-Fe-Al bulk metallic glass. Scr Mater. 2019;167:31-6. https://doi.org/10.1016/j.scriptamat.2019.03.030.

11. Pan J, Ivanov YP, Zhou WH, Li Y, Greer AL. Strain-hardening and suppression of shear-banding in rejuvenated bulk metallic glass. Nature. 2020;578:559-62. http://dx.doi.org/10.1038/ s41586-020-2016-3.

12. Zhou H, Hubek R, Peterlechner M, Wilde G. Two-stage rejuvenation and the correlation between rejuvenation behavior and the boson heat capacity peak of a bulk metallic glass. Acta Mater. 2019;179:308-16. https://doi.org/10.1016/j. actamat.2019.08.040.

13. Guo W, Saida J, Zhao M, Lü S, Wu S. Rejuvenation of Zr-based bulk metallic glass matrix composite upon deep cryogenic cycling. Mater Lett. 2019;247:135-8. https://doi.org/10.1016/j. matlet.2019.03.117.

14. Lou Y, Liu X, Yang X, Ge Y, Zhao D, Wang H, et al. Fast rejuvenation in bulk metallic glass induced by ultrasonic vibration precompression. Intermetallics. 2020;118:106687. https://doi.org/10.1016/j.intermet.2019.106687.

15. Sajjadifar S, Zolfigol MA, Chehardoli GH. Boron sulfuric acid as an efficient heterogeneous catalyst for the synthesis of 1-substituted 1H-1, 2, 3, 4-tetrazoles in polyethylene glycol. Eurasian Chem. Commun. 2020;2:812-8.

16. Ryu W, Yamada R, Saida J. Tailored hardening of $\mathrm{ZrCuAl}$ bulk metallic glass induced by $2 \mathrm{D}$ gradient rejuvenation. NPG Asia Mater. 2020;12:52. http://dx.doi.org/10.1038/s41427-020-02338.

17. Zhang M, Wang YM, Li FX, Jiang SQ, Li MZ, Liu L. Mechanical relaxation-to-rejuvenation transition in a Zr-based Bulk Metallic Glass. Sci Rep. 2017;7:625. http://dx.doi.org/10.1038/s41598017-00768-7.

18. Samavatian M, Gholamipour R, Amadeh AA, Samavatian V. Inherent relation between atomic-level stresses and nanoscale heterogeneity in Zr-based bulk metallic glass under a rejuvenation process. Phys B Condens Matter. 2020;595:412390. https://doi. org/10.1016/j.physb.2020.412390.

19. Samavatian M, Gholamipour R, Amadeh AA, Mirdamadi S. Role of tensile elastostatic loading on atomic structure and mechanical properties of $\mathrm{Zr55Cu} 30 \mathrm{Ni} 5 \mathrm{Al10}$ bulk metallic glass. Mater Sci Eng A. 2019;753:218-23. https://doi.org/10.1016/j. msea.2019.03.058.
20. Samavatian M, Gholamipour R, Amadeh AA, Mirdamadi S. Extra rejuvenation of $\mathrm{Zr55Cu30A110Ni5}$ bulk metallic glass using elastostatic loading and cryothermal treatment interaction. J Non-Cryst Solids. 2019;506:39-45. https://doi.org/10.1016/j. jnoncrysol.2018.12.007.

21. Tong Y, Dmowski W, Bei H, Yokoyama Y, Egami T. Mechanical rejuvenation in bulk metallic glass induced by thermo-mechanical creep. Acta Mater. 2018;148:384-90. https://doi.org/10.1016/j. actamat.2018.02.019.

22. Wang P, Yang X. Atomistic investigation of aging and rejuvenation in $\mathrm{CuZr}$ metallic glass under cyclic loading. Comput Mater Sci. 2020;185:109965. https://doi.org/10.1016/j. commatsci.2020.109965.

23. Li S, Huang P, Wang F. Rejuvenation saturation upon cyclic elastic loading in metallic glass. Comput Mater Sci. 2019;166:318-25. https://doi.org/10.1016/j.commatsci.2019.05.007.

24. Ding G, Li C, Zaccone A, Wang WH, Lei HC, Jiang F, et al. Ultrafast extreme rejuvenation of metallic glasses by shock compression. Sci Adv. 2019;5(8):eaaw6249. http://dx.doi. org/10.1126/sciadv.aaw6249.

25. Sohrabi S, Li MX, Bai HY, Ma J, Wang WH, Greer AL. Energy storage oscillation of metallic glass induced by high-intensity elastic stimulation. Appl Phys Lett. 2020;116:81901. http:// dx.doi.org/10.1063/1.5140208.

26. Fu Y, Wang G, Gao J, Yao Q, Tong W. New approach to produce a nanocrystalline layer on surface of a large size pure titanium plate. Coatings. 2020;10:430.

27. Liu J, Wang Q, Sun K, Gravier S, Blandin J, Sun B, et al. Serrated plastic flow behavior and microstructure in a Zr-based bulk metallic glass processed by surface mechanical attrition treatment. J Iron Steel Res Int. 2017;24:475-82. https://doi. org/10.1016/S1006-706X(17)30072-9.

28. Lee J-C. Calorimetric study of $\beta$-relaxation in an amorphous alloy: an experimental technique for measuring the activation energy for shear transformation. Intermetallics. 2014;44:11620. https://doi.org/10.1016/j.intermet.2013.09.002.

29. Ketov SV, Trifonov AS, Ivanov YP, Churyumov AY, Lubenchenko $\mathrm{AV}$, Batrakov AA, et al. On cryothermal cycling as a method for inducing structural changes in metallic glasses. NPG Asia Mater. 2018;10:137-45. http://dx.doi.org/10.1038/s41427-0180019-4.

30. Zhu F, Nguyen HK, Song SX, Aji DPB, Hirata A, Wang $\mathrm{H}$, et al. Intrinsic correlation between $\beta$-relaxation and spatial heterogeneity in a metallic glass. Nat Commun. 2016;7:11516. http://dx.doi.org/10.1038/ncomms11516.

31. Greer AL, Sun YH. Stored energy in metallic glasses due to strains within the elastic limit. Philos Mag. 2016;96(16):164363. http://dx.doi.org/10.1080/14786435.2016.1177231.

32. Ebner C, Pauly S, Eckert J, Rentenberger C. Effect of mechanically induced structural rejuvenation on the deformation behaviour of CuZr based bulk metallic glass. Mater Sci Eng A. 2020;773:138848. https://doi.org/10.1016/j.msea.2019.138848.

33. Meng F, Tsuchiya K, Kramer MJ, Ott RT. Reduction of shear localization through structural rejuvenation in $\mathrm{Zr}-\mathrm{Cu}-\mathrm{Al}$ bulk metallic glass. Mater Sci Eng A. 2019;765:138304. https://doi. org/10.1016/j.msea.2019.138304.

34. Cao JW, Han JG, Guo ZH, Zhao WB, Guo YQ, Xia ZH, et al. Plasticity enhancement of high-entropy bulk metallic glasses by electroless plating with Ni-P amorphous films. Mater Sci Eng A. 2016;673:141-7. https://doi.org/10.1016/j.msea.2016.07.078.

35. Greer AL, Cheng YQ, Ma E. Shear bands in metallic glasses. Mater Sci Eng R Reports. 2013;74:71-132. https://doi.org/10.1016/j. mser.2013.04.001.

36. Chen SH, Li T, Chang WJ, Yang HD, Zhang JC, Tang HH, et al. On the formation of shear bands in a metallic glass under tailored complex stress fields. J Mater Sci Technol. 2020;53:112-7. https://doi.org/10.1016/j.jmst.2020.02.082. 
37. Li Y, Wei Y, Zhang K, Zhang Y, Wang Y, Tang W, et al. Rejuvenation, embryonic shear bands and improved tensile plasticity of metallic glasses by nanosecond laser shock wave. J Non-Cryst Solids. 2019;513:76-83. https://doi.org/10.1016/j. jnoncrysol.2019.02.031.

38. Kosiba K, Şopu D, Scudino S, Zhang L, Bednarcik J, Pauly S. Modulating heterogeneity and plasticity in bulk metallic glasses: role of interfaces on shear banding. Int J Plast. 2019;119:15670. https://doi.org/10.1016/j.ijplas.2019.03.007.
39. Liu Z, Huang P, Wang F. The correlation between $\beta$ relaxation and shear transformation zone in LaNiAl bulk metallic glasses: the effect of cryogenic thermal cycling treatment. $\mathrm{J}$ Alloys Compd. 2021;865:158993. https://doi.org/10.1016/j. jallcom.2021.158993.

40. Utiarahman A, Alkaim AF, Aljeboree AM, Venediktovna SZ, Takhirovna CO, Alexander M, et al. Role of elastostatic loading and cyclic cryogenic treatment on relaxation behavior of Cebased amorphous alloy. Mater Today Commun. 2021;26:101843. https://doi.org/10.1016/j.mtcomm.2020.101843. 